\title{
Energy and environmental evaluation of combined cooling heating and power system
}

\author{
Andrzej Bugaj ${ }^{1, *}$ \\ ${ }^{1}$ Wroclaw University of Science and Technology, Faculty of Environmental Engineering, \\ ul. Norwida 4/6, 50-373 Wroclaw, Poland
}

\begin{abstract}
The paper addresses issues involving problems of implementing combined cooling, heating and power (CCHP) system to industrial facility with well-defined demand profiles of cooling, heating and electricity. The application of CCHP system in this particular industrial facility is being evaluated by comparison with the reference system that consists of three conventional methods of energy supply: (a) electricity from external grid, (b) heat from gas-fired boilers and (c) cooling from vapour compression chillers run by electricity from the grid. The CCHP system scenario is based on the combined heat and power (CHP) plant with gas turbine-compressor arrangement and water/lithium bromide absorption chiller of a single-effect type. Those two scenarios are analysed in terms of annual primary energy usage as well as emissions of $\mathrm{CO}_{2}$. The results of the analysis show an extent of primary energy savings of the CCHP system in comparison with the reference system. Furthermore, the environmental impact of the CCHP usage, in the form of greenhouse gases emission reductions, compares quite favourably with the reference conventional option.
\end{abstract}

\section{Introduction}

Conventional thermoelectric power plants convert only about $35 \%$ of primary energy into electricity. The rest of primary energy in the form of heat is usually dispersed and can be considered as an energy loss. One of the methods of increasing the efficiency of electricity generation is combined heat and power (CHP) production known also as cogeneration. CHP plant transforms over $80 \%$ of primary energy to usable energy in the form of heat and power. That high conversion efficiency translates also into improved environmental impact giving considerable reduction in emissions of greenhouse gases. The ideal situation is to run a CHP plant throughout the whole year with full utilization of produced heat and power. In Polish climate while the heat from CHP unit is being used in heating, ventilating and air conditioning installations during winter time, afterwards there is always an excess of available heat that is a by-product of electricity generation in the rest of the year. A method of using this excess heat is based on expanding the CHP plant to combined cooling, heating and power generation process also known as tri-generation. The trigeneration or the CCHP system is in fact CHP plant connected to absorption chiller fired

* Corresponding author: andrzej.bugaj@pwr.edu.pl 
with the heat produced in CHP unit. In this way the heat would not be wasted in summer season due to the lack of heat demand but instead it can be used effectively to make cooling energy, e.g. for air conditioning purposes.

CCHP system considered for the particular application in the presented study can use gas reciprocating engines or gas turbines with absorption cooling units. LiBr-water absorption cycle is chosen because the cooling effect is needed mostly in air conditioning installations with cooling water temperatures always above $5^{\circ} \mathrm{C}$. The choice of the most appropriate CCHP system arrangement depends on such factors as heat/power ratio, the temperature level of the required heat output and variations of heating, cooling and power demand.

Numerous literature positions illustrate the specific benefits of using CCHP systems in comparison with conventional alternatives. Apart from a better conversion of primary energy and consequently reduction of greenhouse gases emissions also economic benefits are evident for CCHP options [1]. The same total energy produced in tri-generation process is costs about $30 \%$ less than by conventional ways. The energy supply from the CCHP plant is more reliable than the electricity from the grid. Additionally, the tri-generation units ensure some increase in the electricity grid stability. During hot summer time there would be a significant relief in the grid since cooling process changes from compression to absorption cycles. That further improves efficiency because summer demand peaks are often served by utilities through inefficient stand-by units and/or overloaded transmission lines.

The CCHP system consists of two parts, CHP unit and absorption chiller. There is some variety of these two plants and thus one should choose the appropriate type of the plant for the particular application. Since it is assumed that a continuous power demand prevails on the site and thermal energy can be utilized throughout the year a gas turbine type of CHP is chosen. As for the absorption chiller it is decided to have the LiBr-water unit of two different effect types The first one is a single-effect appliance with small COP value of 0.65 and correspondingly low temperature of medium running the unit. The second one is a two-effect unit with higher COP value of 1.2 and likewise a need of higher temperature to run the chiller.

\section{Case study description}

The case study presented in this paper addresses issues regarding a problem of introducing CCHP system to an industrial facility with well-defined demand of cooling, heating and electricity. The industrial facility is a pharmaceutical factory producing adhesive dressings and plaster materials. The factory is currently being expanded by installing additional production lines. All new production lines should be housed in air conditioned spaces. The production processes are assumed to be run of 16 to 20 hours per day with 5 to 6 days schedules depending on the product demand. Until now air conditioned spaces use heating energy provided by gas fired boilers and cooling energy is supplied from vapour compression type chillers. All electricity needs are covered by external electrical grid. The projected process of production expansion poses a question whether it is worthwhile attitude to increase the existing ways of energy supply that mean additional boilers, larger compression chillers and bigger electricity demand from the grid. Or perhaps one should consider energy production on the site with CCHP system. The most economic operation option is to run the CCHP plant through the year with almost full utilization of produced on site heat and power. 


\subsection{Conventional and CCHP systems}

The application of CCHP system in this particular industrial facility should be assessed in comparison with a conventional option that is a baseline scenario called Case 1. This reference scenario encompasses three standard ways of providing to the site: (a) electricity from the external grid, (b) heat from the gas-fired boilers and (c) cooling from vapour compression chillers run by electricity from the grid, as it is illustrated in fig.1. Electricity taken from the grid is used to cover a power load of production lines and to generate cooling energy in compression chillers. Conversely, natural gas is supplied to the boilers ensuring all heating needs.

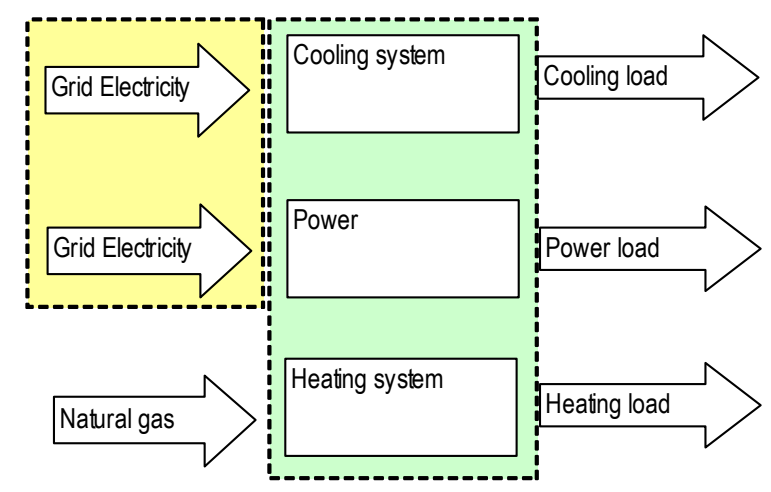

Fig. 1. Conventional system - Case 1 .

The CCHP scenario called Case 2 is based on the operation of the CHP plant producing all needed electricity while recovered heat is being used to generate cooling energy in an absorption chiller and to cover a heating load, as it is illustrated in fig.2.

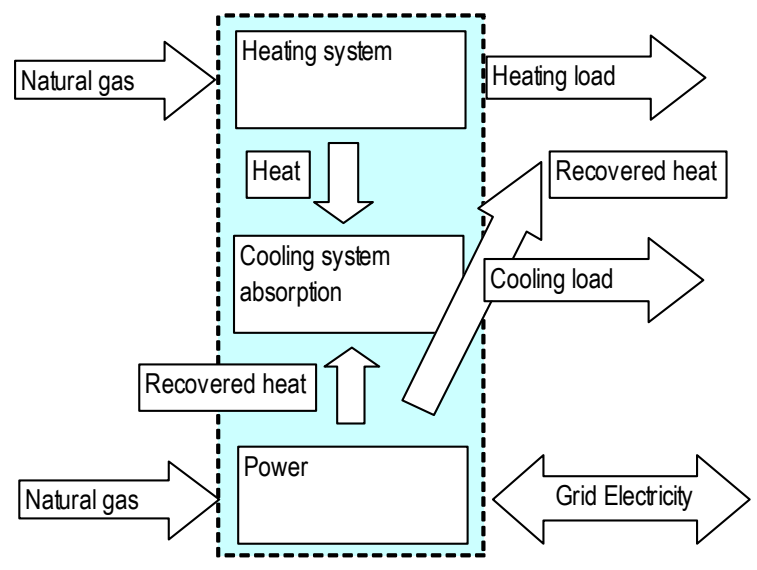

Fig. 2. CCHP system - Case 2 .

In the general practice of using CHP units, occasionally a certain amount of cooling energy could be also generated in vapour compression chillers supplied with electricity 
from the CHP unit. But in the case discussed it is not a viable option because there would be an excess of heat from the CHP unit in summer season. That excess heat could be otherwise wasted and an overall efficiency of the system would decrease considerably. Additionally, the heating system is equipped with gas boilers which could be used as backup.

\section{System performance analyses}

Most of the studies on CCHP system performance relate to energy, environmental and economic evaluations [2,3]. The authors use primary energy savings ratio and $\mathrm{CO}_{2}$ emission reduction ratio to assess the energy usage and environmental impact of the system, respectively. In general, it is well known that CCHP systems are rather difficult to design and operate in facilities with distinctively varying power, cooling and heating demands, as it is a case in residential and commercial buildings. However, some investigations were carried out for these types of buildings and reported in technical literature [4-7]. In contrast, very few researches can be found on the CCHP performance in cases with more uniform power and thermal loads, e.g. in data centres building. A comprehensive research on this type of site is described in [8,9]. This paper appraises the CCHP performance on the industrial site where power, heating and cooling loads fluctuate in different mode than in the separate buildings due to thermal needs of the production lines.

Three elements of the presented study: data patterns, the simulation model and performance evaluation constitute comprehensive procedure for the system operation and efficiency assessment. In addition, a sensitivity parameter analysis can be used to optimize design of the system according to prevailing demand profiles. Data patterns describing power and thermal demands serve as inputs to the simulation model. The model is based on existing and validated procedures of TRANSYS program taking into account equipment specifications, system configurations and operational schedule. This report focuses mostly on using the simulation model results in the process of energy and environmental evaluation of the CCHP system in the similar way like in the approach described in [9].

The primary energy consumption of the system is obtained by multiplying the entire amount of energy consumed on site by its primary energy factor that takes into account all losses occurring throughout conversion, transmission, storage and distribution. Hence, the primary energy consumption is used as the basis for energy performance evaluation. This energy consumption of the conventional system in Case $1, P E_{c o n v}$, is calculated as follows:

$$
P E_{\text {conv }}=\left(E_{e l}+E_{\text {cool }} / C O P_{c}\right) F_{e l}+\left(E_{h b} / \eta_{t}\right) F_{g}
$$

where $E_{e l}$ is the power load integrated over time, $E_{\text {cool }}$ is the cooling energy generated by compression chiller, $C O P_{c}$ is coefficient of performance of the chiller, $E_{h b}$ is the heating load taken as heating energy produced by gas boilers with total efficiency of $\eta_{t}$. $E_{h b}$ can also be interpreted as the amount of energy covering all central heating, ventilation and technological needs. $F_{g}$ and $F_{e l}$ are primary energy factors for gas heating and electricity, respectively. The following values of these factors were taken for the study: $F_{g}=1.1$ and $F_{e l}=3.0$. Consequently, the primary energy consumption of the CCHP system in Case 2 is determined in the following way:

$$
P E_{C C H P}=\left(E_{e l}+E_{\text {cool }} / C O P_{a b s}+E_{h}\right) F_{g}+\left(E_{h b b} / \eta_{t}\right) F_{g}
$$

where $E_{\text {cool }}$ in this case is the cooling energy generated by absorption chiller characterized by $C O P_{a b s}, E_{h}$ is heating energy produced by the CCHP system and $E_{h b b}$ denotes energy taken from the gas backup boilers. 
In addition to the primary energy rating, a carbon dioxide apprising was also performed as stated in the standard EN 15603:2008. The combined environmental impact of all greenhouse gas compounds is commonly normalized to the specific effect of $\mathrm{CO}_{2}$ and all emissions are expressed in $\mathrm{CO}_{2}$ equivalents. For the purpose of this study the emissions are just expressed in the mass of $\mathrm{CO}_{2}$. Emission factors for gas usage, $E F_{g}$ and electricity production, $E F_{e}$ representative in the local energy market were introduced in order to calculate actual emissions. The following values are currently used in Poland: $E F_{g}=0.202 \mathrm{kgCO}_{2} / \mathrm{kWh}$ and $E F_{e}=0.812 \mathrm{~kg} \mathrm{CO} / \mathrm{kWh}$. The annual $\mathrm{CO}_{2}$ emissions for the conventional system of the Case $1\left(A E_{\text {conv }}\right)$ are computed in the following way:

$$
A E_{\text {conv }}=\left(E_{e l}+E_{\text {cool }} / C O P_{c}\right) E F_{e}+\left(E_{h b} / \eta_{t}\right) E F_{g}
$$

Consequently, for the CCHP system of the Case 2 the annual $\mathrm{CO}_{2}$ emissions are found from the following formula:

$$
A E_{C C H P}=\left(E_{e l}+E_{c o o l} / C O P_{a b s}+E_{h}\right) E F_{g}+\left(E_{h b b} / \eta_{t}\right) E F_{g}
$$

\section{Results and discussion}

The foremost task in the process of optimizing the CCHP system performance is to match its operation with existing heating, cooling and power load profiles. Fig.3 shows the monthly power demands as well as heating and cooling loads occurring on site.

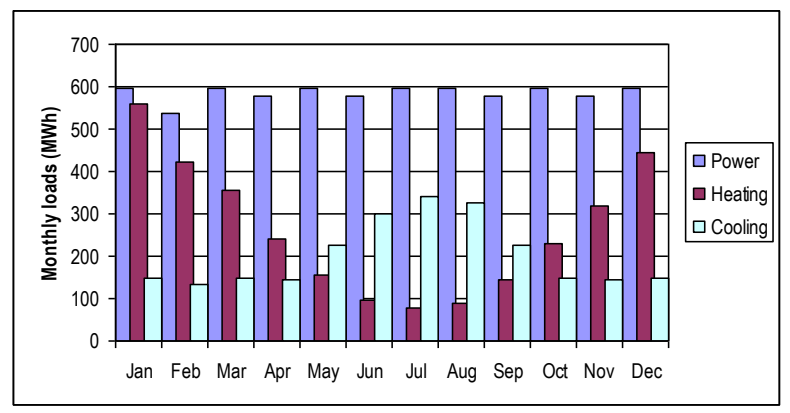

Fig. 3. Power, cooling and heating monthly loads.

Monthly load profiles of all three forms of energy shown in Fig.4 illustrate a relation between the profiles throughout a year.

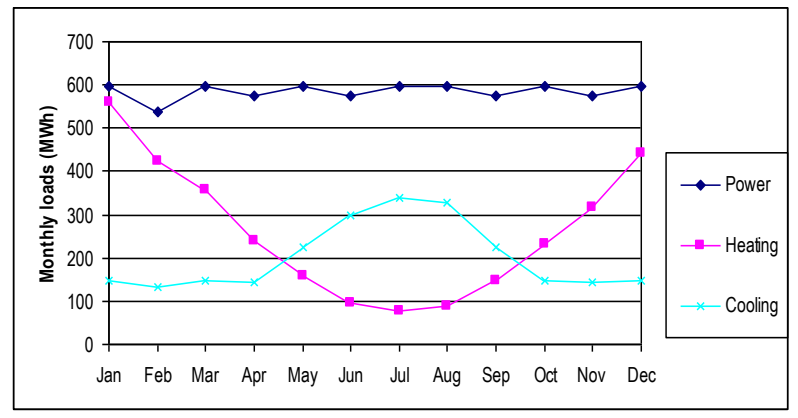

Fig. 4. Annual power, cooling and heating profiles. 
Maximum heating needs occur during winter months but heating is also required during summer mostly due to production lines demand. The cooling energy is not only required in summer for air conditioning installations but there is also quite a considerable demand of cooling for manufacturing purposes. On the whole, all energy load profiles on site look rather suitable for the usage of the CCHP system.

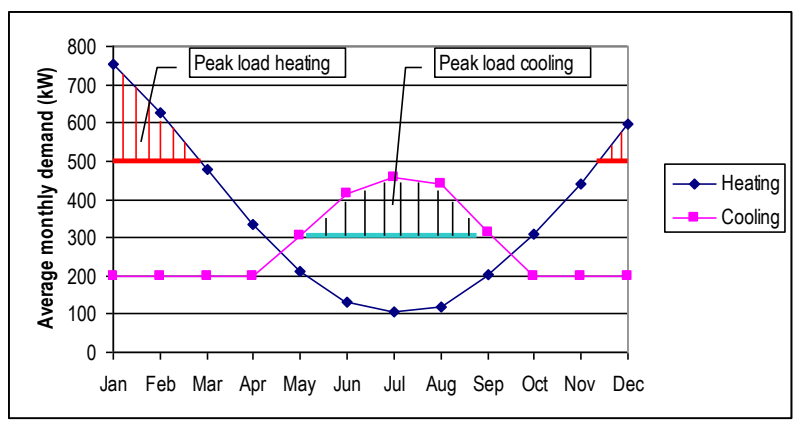

Fig. 5. Average monthly demand of heating and cooling.

However, the problem with the instantaneous capacity demands can take place when production and HVAC needs (expressed in $\mathrm{kW}$ ) attain their maximum values at the same time resulting in either peak load heating or peak load cooling as shown in Fig.5.

Fig.6 shows how the CCHP system can adapt to the monthly power, heating and cooling loads of the facility. The total heating load in the form of heat recovered from the CCHP system is increased at that moment to the value covering the heating and cooling needs. The cooling energy is then generated by heat in the absorption chiller.

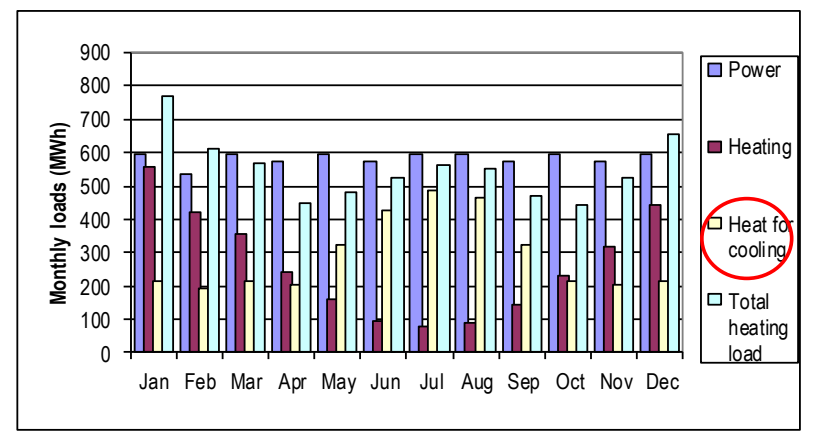

Fig. 6. Monthly loads of power, heating and cooling - Case 2.

The relation between all four load profiles in a yearly framework is presented in Fig. 7. Fig. 8 depicts entire annual primary energy consumption for the conventional system of Case 1 and for the CCHP system of Case 2. This total primary energy is additionally allocated to the specific installations such as power, heating and cooling systems. The usage of total primary in the CCHP system is $24 \%$ smaller than in the conventional system. In power utilization the reduction in Case 2 versus Case 1 ranges up to $32 \%$. Alternatively, in heating production Case 2 gives an increase of 55\% in comparison with Case 1 . In contrast, cooling generation in Case 2 offers primary energy reduction of $67 \%$ as opposed to Case 1. 


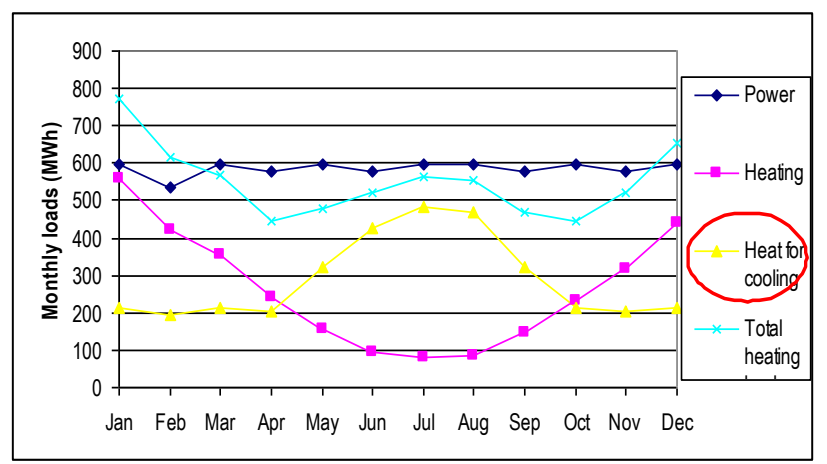

Fig. 7. Annual power, cooling and heating profiles - Case 2 .

Fig. 9 illustrates annual $\mathrm{CO}_{2}$ emissions for Case 1 of the conventional system and Case 2 of the CCHP system. The total $\mathrm{CO}_{2}$ emissions are similarly assigned to power, heating and cooling installations. The total annual emissions in the CCHP system is around $36 \%$ lower than in the conventional system. The emission reduction in power utilization in Case 2 is just $7 \%$ lower than in Case 1.

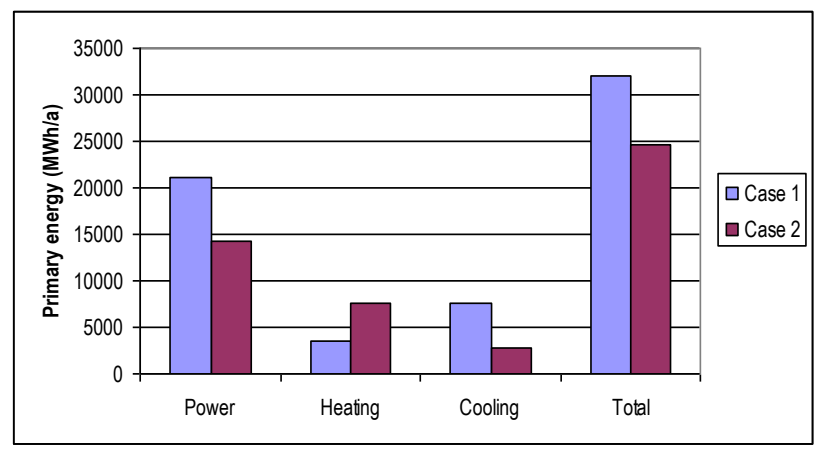

Fig. 8. Primary energy consumption - Case 1 and Case 2 .

Alternatively, in heating energy production the emission reduction in Case 2 reaches $90 \%$ in contrast to Case 1. Furthermore, cooling generation in Case 2 offers no $\mathrm{CO}_{2}$ emission.

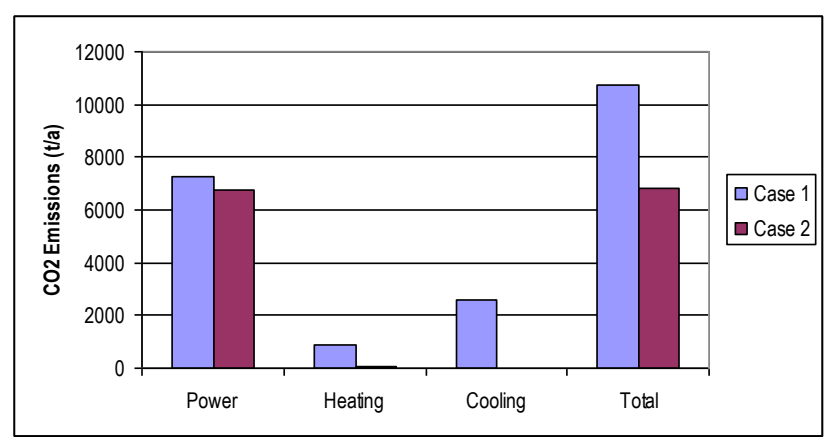

Fig. 9. Annual $\mathrm{CO}_{2}$ emissions - Case 1 and Case 2. 


\section{Conclusions}

This paper presents an energy and environmental evaluation of the CCHP system in the context of a case study with the conventional system in the industrial facility. According to some evaluation results the following conclusions can be presumed. (1) A relatively stable power demand and technological cooling and heating needs associated with the HVAC loads can match in quite favourable way with the CCHP system operation. Therefore, the industrial facility could be rather suitable site for CCHP applications. (2) The energy performance assessment based on the comparison of primary energy consumption in two systems (conventional and CCHP) indicates that the CCHP system attains better performance than the conventional one. (3) Likewise, the environmental performance appraisal based on the total annual $\mathrm{CO}_{2}$ emissions proves that the CCHP system is more environmentally benign than the conventional one. (4) Additionally, there also exist several other opportunities to optimize the operation of the proposed CCHP system in this particular industrial facility. One of these measures could be a replacement of a single effect absorption unit with a two-effect unit with increasing effectiveness of cooling generation almost twofold. Another operation optimizing measure would an improvement of demand side management on site, especially with grid electricity and backup boilers.

\section{References}

1. M. Ebrahimi, A. Keshavarz, Combined cooling, heating and power (Elsevier, 2015)

2. H. Li, L. Fu, K. Geng, Y. Jiang, Energy and Buildings 38, 253-257 (2006)

3. F. Fang, L. Wei, J. Liu, J. Zhang, Energy 46, 211-220 (2012)

4. P,J, Mago, L.M. Chamra, Energy and Buildings 41, 1099-1106 (2009)

5. M. Carvalho, L.M. Serra, M.A. Lozano, Energy 36, 3779-3790 (2011)

6. Q. Gu, H. Ren, W. Gao, J. Ren, Energy and Buildings 51, 143-152 (2012)

7. G. Pagliarini, C. Corradi, S. Rainieri, Appl. Therm. Eng. 44, 150-158 (2012)

8. G.L. Guizzi, M. Manno, M. Kuosa, Energy 41, 56-64 (2012)

9. D. Xu, M. Qu, Energy and Buildings 67, 176-186 (2013) 